\title{
Single- and Double-Lung Ventilation in Infants and Children Undergoing Thoracoscopic Lung Resection
}

\author{
Carmen Dingemann ${ }^{1}$ Christoph Zoeller ${ }^{1}$ Ziad Bataineh $^{1}$ Alexander Osthaus ${ }^{2}$ \\ Robert Suempelmann ${ }^{2}$ Benno Ure ${ }^{1}$
}

${ }^{1}$ Department of Pediatric Surgery, Hannover Medical School, Hannover, Germany

2 Department of Anaesthesiology, Hannover Medical School, Hannover, Germany

Address for correspondence and reprint requests Dr. Carmen Dingemann, Department of Pediatric Surgery, Hannover Medical School, Carl-Neuberg-Strasse 1, Hannover 30625, Germany (e-mail: dingemann.carmen@mh-hannover.de).

Eur J Pediatr Surg 2013;23:48-52.

\begin{abstract}
Keywords

- single-lung ventilation

- thoracoscopy

- children

- lung resection

- pediatric surgery

Introduction Video-assisted thoracoscopic surgery (VATS) has gained wide acceptance for the pediatric population. Single-lung ventilation (SLV) has been suggested for thoracoscopic lung resection to provide better surgical exposure, but its role and sequelae compared with double-lung ventilation (DLV) have not been determined. The aim of this study was to investigate the feasibility and effects of SLV and DLV in infants and children undergoing thoracoscopic lung resection.

Patients and Methods Written informed consent from all guardians for anonymized data analysis and approval by the Institutional Review Board were obtained. A retrospective study on a consecutive series of infants and children who underwent thoracoscopic lung resection during an 11 years period was performed. SLV was selected mainly in lesions localized in the upper, middle, and/or central lung for reasons of surgical exposure. Patients with lower lobe lesions and those who underwent atypical resections were preferably operated under DLV. End points were conversion rate, duration of postoperative ventilation, and perioperative complications, such as, atelectasis or pneumonia.

Results Of 114 pediatric patients (58 female and 56 male; ratio 1.04:1) with a mean age of 7.1 years ( 3 days to 18.1 years), 62 patients underwent DLV and 52 patients underwent SLV for thoracoscopic lung resection. There were no significant differences between the two groups for conversion rate (DLV 8.1 vs. SLV 6.1\%; $p=0.53$ ), prompt extubation (DLV 50 vs. SLV 34.6\%; $p=0.14$ ), and postoperative atelectasis (DLV 35.5 vs. SLV 25\%; $p=0.32$ ). No major cardiorespiratory events, such as bleeding or pneumonia, were observed. No perioperative mortality occurred.

Conclusions This is the first study on safety, effectiveness, and outcome of SLV and DLV in pediatric patients undergoing thoracoscopic lung resection. Our data suggest that both SLV and DLV can be safely performed with similar low rate of surgical complications, when specific selection criteria are applied.
\end{abstract}

received

May 16, 2012

accepted

June 27, 2012

published online

October 23, 2012 (c) 2013 Georg Thieme Verlag KG

Stuttgart · New York
DOI http://dx.doi.org/ 10.1055/s-0032-1324693. ISSN 0939-7248. 


\section{Introduction}

Video-assisted thoracoscopic surgery (VATS) has gained wide acceptance in pediatric thoracic surgery. ${ }^{1-6}$ Numerous studies have demonstrated advantages of VATS versus conventional surgery, such as lower postoperative pain, lower morbidity, faster recovery, superior cosmetic results, and significantly less musculoskeletal sequelae. ${ }^{1-3,7}$ Safety and efficacy of VATS in children have also been confirmed. ${ }^{1,4,8,9}$

Ipsilateral pulmonary ventilation might jeopardize the success of VATS due to poor surgical exposure and might result in conversion to open surgery. Therefore, single-lung ventilation (SLV) has been introduced to improve the feasibility of VATS. Various SLV techniques have been assessed for adult patients, ${ }^{10-15}$ and several studies have confirmed the feasibility and safety of SLV in VATS in neonates and children. $3,4,16,17$

However, the indications for SLV in infants and children have not been clearly determined yet, ${ }^{18,19}$ and the role of SLV in thoracoscopic lung resection remains to be clarified. SLV has been postulated to be absolutely indicated when the nondiseased lung must be protected from ipsilateral lung pathology, such as, hemorrhage, infection, or spillage of tumor cells. Historically, VATS was considered to be an absolute indication for SLV to provide surgical exposure in adult patients. ${ }^{10,12-14}$ More recently, conventional double-lung ventilation (DLV) has proven to be efficient as well, so that now VATS is considered to be a relative indication for SLV. ${ }^{2,3}$

Herein, we report our experience with SLV and DLV in a consecutive series of pediatric patients, who underwent thoracoscopic lung resection with regard to safety, effectiveness, and outcome. We hypothesized that the feasibility of SLV is excellent, but that DLV in selected patients undergoing lung resection leads to similar results.

\section{Patients and Methods}

\section{Patients}

The study was approved by the Institutional Review Board (approval number: 995-2011). Written informed consent was obtained from all patients/guardians for anonymized data analysis.

We reviewed the medical records of all consecutive pediatric patients, who underwent lung resection at our tertiary referral center during an almost 11 -year period (January 2000 to November 2010). Demographical data, type of ventilation, localization, and the extent of lung resection were analyzed. End points of the study were conversion rate of VATS and reasons for conversion, duration of postoperative ventilation, and intra- and postoperative complications, such as, atelectasis or pneumonia.

Atelectasis was defined by sharply lined areas of volumedecreased, radio-opaque lung fields on chest X-ray. Pneumonia was defined by opacity of lung fields requiring antibiotic therapy due to symptoms, such as, fever, and associated laboratory findings. ${ }^{20}$ The diagnosis of atelectasis, pneumonia, and/or other pulmonary pathology was established by a pediatric radiologist.
All lesions were preoperatively evaluated by computed tomography or magnetic resonance imaging. SLV was mainly chosen for reasons of surgical exposure in those cases where the pulmonary lesion was located in the upper, middle, and/ or central lung. Patients with a lesion in the lower lobe and those who underwent atypical resections were preferably operated using DLV. The selection criteria for SLV are summarized in -Table 1.

\section{Anesthesiological Approach}

All operations were performed under general anesthesia. Midazolam was given as an oral premedication 20 minutes prior to anesthesia induction. After intravenous induction with propofol, fentanyl, and rocuronium, patients were intubated endotracheally and ventilated mechanically with sevoflurane in oxygen and air.

SLV was achieved by fiberoptic bronchoscopic guidance using a tracheal intubation fiberscope (Karl Storz, Tuttlingen, Germany) and three different age-adapted techniques with either a narrow 2.2-mm insertion tube (LF-P, Olympus, Tokyo, Japan) or a 3.1-mm insertion tube (LF-DP, Olympus, Tokyo, Japan). Endobronchial intubation using a conventional singlelumen endotracheal tube (SLET) that was inserted into the main-stem bronchus of the dependent lung was performed in patients aged 6 years or younger ( - Table 2 ). In children aged 6 to 12 years, a Univent tube (Fuji Systems Corporation, Tokyo, Japan) was used and the attached bronchus blocker was blocked in the main-stem bronchus of the dependent lung. Children aged 12 years or older were intubated with either a Univent tube, a double-lumen endotracheal tube (DLET; Broncho-Cath; Mallinckrodt Medical, Athlone, Ireland), or an Arndt endobronchial blocker (Cook Medical, Limerick, Ireland). Univent tubes and DLET were changed to SLET postoperatively when prolonged mechanical ventilation was required.

The respiratory rate was increased up to 30 to 50 breaths per minute, and tidal volume was decreased to improve the surgical visualization during artificial pneumothorax. Standard perioperative monitoring included pulse oximetry, electrocardiogram, end-tidal $\mathrm{CO}_{2}$ measurement, inhaled volatile agent concentration, invasive blood pressure measurement, arterial blood gas measurement, and temperature. Extended

Table 1 Preference for Single-Lung Ventilation Based on the Following Standardized Selection Criteria

\begin{tabular}{|l|l|}
\hline Selection Criterion & \\
\hline Patient's weight & $\geq 2000 \mathrm{~g}$ \\
\hline Patient's cardiorespiratory & $\begin{array}{l}\text { No major cardiac anomaly } \\
\text { No cardiac medication } \\
\text { No primary dependence on } \\
\text { mechanical ventilator } \\
\text { No pathology of the } \\
\text { contralateral lung }\end{array}$ \\
\hline Location of pulmonary & $\begin{array}{l}\text { Upper lobe } \\
\text { Middle lobe } \\
\text { lesion }\end{array}$ \\
\hline Type of lung pathology lung (all lobes) \\
\hline
\end{tabular}


Table 2 Age-Adapted Tube Selection for Single-Lung Ventilation in 52 Pediatric Patients Undergoing Thoracoscopic Lung Resection

\begin{tabular}{|l|l|l|l|l|}
\hline Age, $y$ & $\begin{array}{l}\text { SLET, } \\
\text { ID in } \mathbf{m m}\end{array}$ & Univent Tube, ID in mm & $\begin{array}{l}\text { Endobronchial } \\
\text { Blocker, Fr }\end{array}$ & DLET, Fr \\
\hline$\leq 6$ & $3.5-5.5$ & - & - & - \\
\hline $6-12$ & - & $3.5-4.5$ & - & - \\
\hline$\geq 12$ & - & $4.5-7.0$ & 9 & $26-35$ \\
\hline
\end{tabular}

Note: Children of the same age may significantly vary in overall size and the dimensions of the airway. ${ }^{1}$ Larger DLETs may safely be used in teenagers. SLET, single-lumen endotracheal tube; ID, internal diameter; Fr, French size; DLET, double-lumen endotracheal tube.

monitoring was indicated if hemodynamic alterations were anticipated.

\section{Surgical Approach}

All thoracoscopic interventions were performed with the patient in a lateral decubitus position and prepared for open thoracotomy should this have proved necessary. Procedures were performed using reusable instruments (Karl Storz, Tuttlingen, Germany). A video thoracoscope was inserted to visualize the operative field. Carbon dioxide was insufflated to a maximum pressure of 5 to $8 \mathrm{~mm} \mathrm{Hg}$ at a maximum flow rate of $5 \mathrm{~L} / \mathrm{min}$. To prevent $\mathrm{CO}_{2}$-overinsufflation, insufflators specifically suitable for neonates were used, delivering $\mathrm{CO}_{2}$ in small controlled puffs, allowing a better adjustment of the intrathoracic pressure. Three to five valved ports (3.5 to 10 $\mathrm{mm}$ ) were inserted depending on the localization of the pathology. Instruments and surgical technique varied depending on the effected lobe and type of pathology. Lobectomy and segmentectomy were performed using Endostapler (Ethicon, Norderstedt, Germany) or LigaSure Vessel Sealing System (Valleylab, Boulder, Colorado, United States), respectively. Atypical lung resections were performed using Endoloop Ligatures (Ethicon, Norderstedt, Germany) or Endostapler (Ethicon, Norderstedt, Germany).

\section{Statistical Analysis}

Data were quoted as median and interquartile ranges. The statistical software package SPSS version 18 (SPSS, Inc., Chicago, Illinois) was used. Intergroup comparison was assessed using unpaired $t$ test, Mann-Whitney $U$ tests, or chisquare test where appropriate. A $p$ value of less than 0.05 was considered significant.

\section{Results}

During the study period, 114 consecutive patients ( 58 female and 56 male; ratio 1.04:1) with a mean age of 7.1 years (3 days to 18.1 years) underwent thoracoscopic lung resection. The mean body weight and height were $27 \mathrm{~kg}$ (2 to $82 \mathrm{~kg}$ ) and $114.3 \mathrm{~cm}$ (39 to $213 \mathrm{~cm}$ ), respectively (- Table 3 ). Therapeutic lung resection was performed for congenital cystic adenomatoid malformation ( $n=9$ ), bronchopulmonary sequestration $(n=10)$, bronchiectasis $(n=10)$, bronchogenic cyst $(n=7)$, congenital lobar emphysema $(n=18)$, and malignancy (e.g., bronchial carcinoid; $n=9$ ).

Atypical resection was performed for diagnostic purposes $(n=35)$ or for excision of pulmonary metastasis $(n=16)$. Resections were performed for lesions located in the upper lobe in 43 patients (37.7\%), middle lobe in 8 patients (7.0\%), lower lobe in 53 patients (46.5\%), and others in 10 patients (8.8\%) (-Table 4).

Of 114 procedures, 62 procedures (54.4\%) were performed using conventional DLV, whereas 52 procedures (45.6\%) were performed using SLV (-Table 3 ).

Segmentectomy or lobectomy was performed in 61 patients (54\%), of whom, 25 patients (41\%) underwent DLV and 36 patients (59\%) underwent SLV. Atypical lung resections were performed in 53 patients (46\%), of whom, 37 patients (70\%) received DLV and 16 patients (30\%) received SLV.

In the group of patients who underwent SLV, there were 10 of 52 patients (19.2\%) below 1 year of age and 13 of 52 patients (25\%) below $10 \mathrm{~kg}$ body weight.

In the group of patients below 1 year of age who underwent SLV, the operative diagnoses were congenital cystic adenoid malformations or pulmonary sequestration $(n=5)$, bronchogenic cyst $(n=2)$, congenital lobar emphysema, bronchiectasis, or interstitial lung disease $(n=3)$. In nine of these patients, a lobectomy was performed and one patient received an atypical lung resection.

The conversion rate in DLV patients was 5 of 62 (8.1\%). These thoracoscopic operations were converted due to exposure difficulties ( $n=3)$, insufficient ventilation $(n=1)$, and technical problems $(n=1)$ ( $n$ Fig. 1$)$. Seven of 52 SLV procedures $(6.1 \%)$ were converted to an open procedure due to

Table 3 Demographical Data of 114 Infants and Children Who Underwent Thoracoscopic Lung Resection

\begin{tabular}{|l|l|l|l|}
\hline & DLV $(\boldsymbol{n}=62)$ & SLV $(\boldsymbol{n}=52)$ & $p$ Value \\
\hline Age & $5.8 \mathrm{y}(21 \mathrm{~d}-17.8 \mathrm{y})$ & $11.4 \mathrm{y}(3 \mathrm{~d}-18.1 \mathrm{y})$ & 0.013 \\
\hline Gender & 47 female, 15 male & 11 female, 41 male & $<0.01$ \\
\hline Weight, kg & $21.5(3-82)$ & $33.6(2-74)$ & 0.004 \\
\hline Height, $\mathrm{cm}$ & $104.6(47-188)$ & $125.9(39-213)$ & 0.01 \\
\hline
\end{tabular}

DLV, double-lung ventilation; SLV, single-lung ventilation. 
Table 4 Localization of Thoracoscopic Lung Resection in 114 Pediatric Patients

\begin{tabular}{|l|l|l|l|}
\hline Site of Resection & $\begin{array}{l}\text { Number of Patients } \\
\text { Using DLV } \\
(\boldsymbol{n}=62), \boldsymbol{n}(\%)\end{array}$ & $\begin{array}{l}\text { Number of Patients } \\
\text { Using SLV } \\
(\boldsymbol{n}=52), \boldsymbol{n}(\%)\end{array}$ & $\boldsymbol{p}$ Value \\
\hline Upper lobe & $19(30.6)$ & $24(46.2)$ & n.s. \\
\hline Middle lobe & $4(6.5)$ & $4(7.7)$ & n.s. \\
\hline Lower lobe $^{\text {Others }}{ }^{\text {a }}$ & $31(50)$ & $22(42.3)$ & n.s. \\
\hline
\end{tabular}

Note: No statistical analysis was performed due to differences in patient characteristics.

ailateral lower lobe $(n=1)$, upper + lower lobe $(n=1)$, middle + upper lobe $(n=1)$, middle + lower lobe $(n=1)$, upper lobe + mediastinum $(n=5)$, lower lobe + diaphragm $(n=1)$.

DLV, double-lung ventilation; SLV, single-lung ventilation.

exposure difficulties $(n=6)$ and bleeding $(n=1)$. Two of these patients were infants, and both had to be converted due to exposure difficulties. However, none of the infants were converted for hemodynamic pertubation induced by VATS. The conversion rate was not significantly different between the groups $(p=0.53)$.

At the end of the operation, 31 DLV patients (50\%) were immediately extubated, whereas 21 (33.9\%) remained ventilated up to 24 hours postoperatively, and 10 patients (16.1\%) required more than 24 hours of ventilation (-Fig. 2). Conversely, 18 SLV patients (34.6\%) were extubated immediately after surgery, 27 (51.9\%) were extubated within 24 hours, and 7 (13.5\%) were extubated after 24 hours, postoperatively. There were no statistical differences in the duration of postoperative ventilation between the two groups (-Fig. 2).

The mean stay on the intensive care unit after DLV was 4 days ( 0 to 53 days) days compared with 3.3 days ( 0 to 64 days) after SLV $(p=0.52)$. Twenty-two patients $(35.5 \%)$ developed atelectasis after DLV. In all of these patients, atelectasis resolved spontaneously without intervention (-Fig. 1). Following SLV, atelectasis developed in 13 patients (25\%), of whom, one patient required bronchoscopic intervention. The

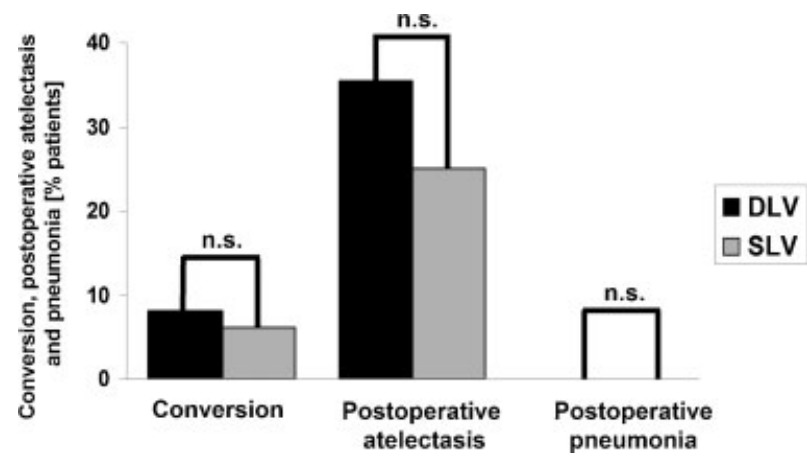

Figure 1 Conversion rate of VATS to open thoracotomy and incidence of postoperative atelectasis and pneumonia in 114 pediatric patients undergoing thoracoscopic lung resection using DLV $(n=62)$ or SLV $(n=52)$. VATS, video-assisted thoracoscopic surgery; DLV, double-lung ventilation; SLV, single-lung ventilation, n.s., not significant. incidence of atelectasis was not significantly different between the groups $(p=0.32)$. Postoperatively, no pneumonia, cardiorespiratory event, or bleeding requiring transfusion occurred (-Fig. 1). There was no perioperative mortality.

\section{Discussion}

Single-lung ventilation during VATS is recommended to create space for adequate visualization, exposure, and dissection while oxygenation is maintained. ${ }^{12,15,18,21}$ Rothenberg postulated that most children can tolerate SLV without significant respiratory compromise during major thoracoscopic procedures., ${ }^{8,9}$ Recently, a study from our institution demonstrated that SLV is feasible and efficient for a broad spectrum of thoracoscopic procedures in children and adolescents and has a low complication rate. ${ }^{17}$ On the contrary, McGahren et al reported that thoracoscopic surgery was successfully performed under DLV in 44 of 68 children. Infants and small children often did not tolerate SLV. ${ }^{22}$ As a consequence, the feasibility of SLV in infants and small children may be limited and SLV might not be necessary for every thoracoscopic operation.

In this series, more than half of the thoracoscopic lung resections were performed using DLV. SLV was more

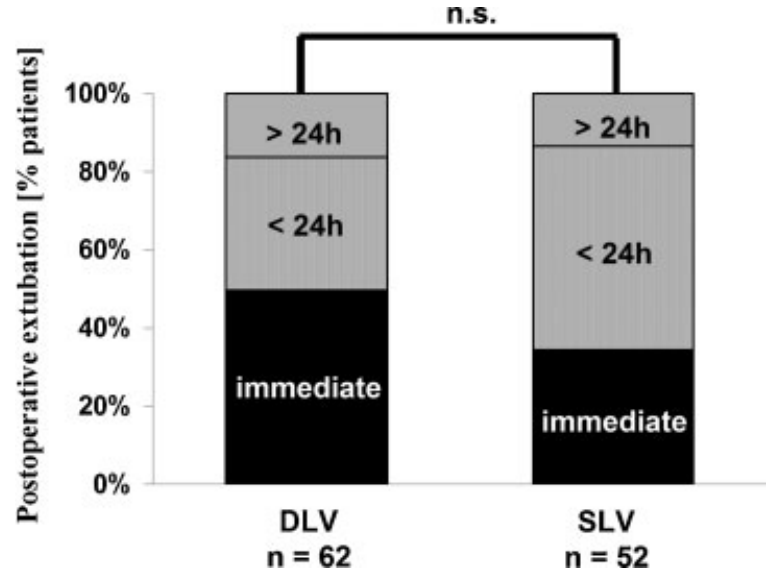

Figure 2 Postoperative extubation in 114 pediatric patients undergoing thoracoscopic lung resection. VATS, video-assisted thoracoscopic surgery; DLV, double-lung ventilation; SLV, single-lung ventilation, n.s., not significant. 
frequently applied when patients were older and in cases in whom the region of interest was located in the upper, middle, and/or central lung. Conventional DLV was preferred in cases where the lesion was located in the lower lobe. In addition, SLV was performed more frequently in extended lung resections, whereas DLV was used more often in atypical resections.

Using this policy, the conversion rate was low and the incidence of complications was similar in both groups. We confirmed an excellent feasibility of VATS, not only in SLV patients, but also in DLV patients. The incidence of problems of visualization and exposure leading to conversion was not significantly different between the groups. Therefore, a recommendation for routine use of SLV in all infants and children undergoing thoracoscopic lung resection cannot be given. Minimally invasive lung resection with DLV may achieve excellent results in a selected group of patients. Nevertheless, the feasibility of SLV was excellent without clinically relevant perioperative complications in our series.

It has been reported that compression of the dependent lung in the lateral decubitus position may cause atelectasis in children. In contrast to adult patients, this is due to several specific factors such as ventilation/perfusion mismatch, reduced hydrostatic pressure gradient between the nondependent and dependent lungs, or the more compliant chest wall. ${ }^{18,23}$ All thoracoscopic procedures in this study were performed with the patient in a lateral decubitus position, which may explain the high incidence of atelectasis after both ventilation strategies. However, bronchoscopic intervention was necessary only in one child of our series and there was no postoperative pneumonia.

A drawback of our study is that the groups of patients who underwent SLV and DLV were not comparable. SLV patients were significantly older and had a higher weight, and the types of operations were not equally distributed.

\section{Conclusions}

This study evaluated safety, effectiveness, and outcome of SLV and DLV in pediatric patients who underwent lung resection. Our data suggest that both SLV and DLV can be safely performed without respiratory compromise or surgical complication, when specific selection criteria are applied. We advocate a differentiated use of both strategies on infants and children undergoing thoracoscopic lung resection.

\section{Conflict of Interest}

None

\section{References}

1 Ure BM, Schmidt AI, Jesch NK. Thoracoscopic surgery in infants and children. Eur J Pediatr Surg 2005;15(5):314-318

2 Byon HJ, Lee JW, Kim JK, et al. Anesthetic management of videoassisted thoracoscopic surgery (VATS) in pediatric patients: the issue of safety in infant and younger children. Korean J Anesthesiol 2010;59(2):99-103

3 Haynes SR, Bonner S. Review article: anaesthesia for thoracic surgery in children. Paediatr Anaesth 2000;10(3):237-251

4 Gentili A, Lima M, De Rose R, Pigna A, Codeluppi V, Baroncini S. Thoracoscopy in children: anaesthesiological implications and case reports. Minerva Anestesiol 2007;73(3):161-171

5 Holcomb GW III, Rothenberg SS, Bax KM, et al. Thoracoscopic repair of esophageal atresia and tracheoesophageal fistula: a multi-institutional analysis. Ann Surg 2005;242(3):422-428, discussion 428-430

6 Adzick NS, Nance ML. Pediatric surgery. First of two parts. N Engl J Med 2000;342(22):1651-1657

7 Lawal TA, Gosemann JH, Kuebler JF, Glüer S, Ure BM. Thoracoscopy versus thoracotomy improves midterm musculoskeletal status and cosmesis in infants and children. Ann Thorac Surg 2009;87 (1):224-228

8 Rothenberg SS. First decade's experience with thoracoscopic lobectomy in infants and children. J Pediatr Surg 2008;43 (1):40-44, discussion 45

9 Rothenberg SS. Experience with thoracoscopic lobectomy in infants and children. J Pediatr Surg 2003;38(1):102-104

10 Fischer GW, Cohen E. An update on anesthesia for thoracoscopic surgery. Curr Opin Anaesthesiol 2010;23(1):7-11

11 Brodsky JB. Lung separation and the difficult airway. Br J Anaesth 2009;103(Suppl 1):i66-i75

12 Cohen E. Methods of lung separation. Curr Opin Anaesthesiol 2002;15(1):69-78

13 Benumof JL. Indication of one-lung anesthesia. In: Miller RD, ed. Anesthesia. New York, NY: Churchill Livingstone; 1992:1689-1690

14 Slinger PD. Lung isolation. In: Young JA, ed. Cardiac Vascular and Thoracic Anesthesia. New York, NY: Churchill Livingstone; 2000: 603-605

15 Brodsky JB, Fitzmaurice B. Modern anesthetic techniques for thoracic operations. World J Surg 2001;25(2):162-166

16 Choudhry DK. Single-lung ventilation in pediatric anesthesia. Anesthesiol Clin North America 2005;23(4):693-708, ix

17 Bataineh ZA, Zoeller C, Dingemann C, Osthaus A, Suempelmann R, Ure B. Our experience with single lung ventilation in thoracoscopic paediatric surgery. Eur J Pediatr Surg 2012;22(1):17-20

18 Means LJ, Green MC, Bilal R. Anesthesia for minimally invasive surgery. Semin Pediatr Surg 2004;13(3):181-187

19 Levine M, Slinger P. Single-lung ventilation in pediatrics. Can J Anaesth 2002;49(3):221-225

20 Hansell DM, Bankier AA, MacMahon H, McLoud TC, Müller NL, Remy J. Fleischner Society: glossary of terms for thoracic imaging. Radiology 2008;246(3):697-722

21 Dave N, Fernandes S. Anaesthetic implications of paediatric thoracoscopy. J Minim Access Surg 2005;1(1):8-14

22 McGahren ED, Kern JA, Rodgers BM. Anesthetic techniques for pediatric thoracoscopy. Ann Thorac Surg 1995;60(4):927-930

23 Hammer GB. Single-lung ventilation in infants and children. Paediatr Anaesth 2004;14(1):98-102 